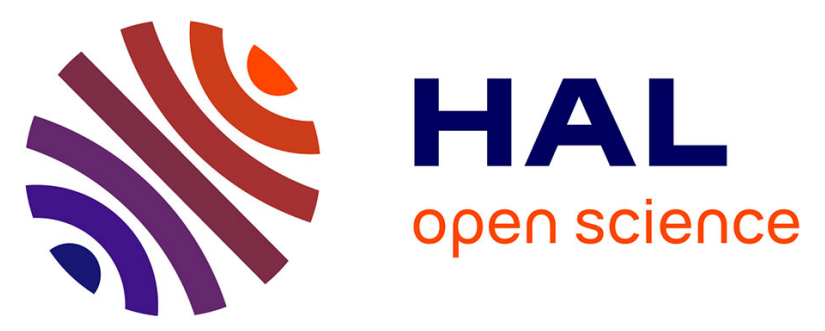

\title{
Fingerprinting mean composition of lithium polysulfide standard solutions by applying high energy resolution fluorescence detected X-ray Absorption Spectroscopy
}

\author{
A. Robba, C. Barchasz, K. Bučar, M. Petric, M. Žitnik, K. Kvashnina, G. \\ Vaughan, R. Bouchet, F. Alloin, M. Kavčič
}

\section{To cite this version:}

A. Robba, C. Barchasz, K. Bučar, M. Petric, M. Žitnik, et al.. Fingerprinting mean composition of lithium polysulfide standard solutions by applying high energy resolution fluorescence detected X-ray Absorption Spectroscopy. Journal of Physical Chemistry Letters, 2020, 11, pp.5446-5450. 10.1021/acs.jpclett.0c01120 . hal-02891524

\section{HAL Id: hal-02891524 \\ https://hal.science/hal-02891524}

Submitted on 6 Jul 2020

HAL is a multi-disciplinary open access archive for the deposit and dissemination of scientific research documents, whether they are published or not. The documents may come from teaching and research institutions in France or abroad, or from public or private research centers.
L'archive ouverte pluridisciplinaire HAL, est destinée au dépôt et à la diffusion de documents scientifiques de niveau recherche, publiés ou non, émanant des établissements d'enseignement et de recherche français ou étrangers, des laboratoires publics ou privés. 


\section{Fingerprinting mean composition of lithium}

\section{polysulfide standard solutions by applying high energy resolution fluorescence detected X-ray}

\section{Absorption Spectroscopy}

A. Robba ${ }^{a, b}$, C. Barchasz ${ }^{a,{ }^{*}}$, K. Bučar ${ }^{d}$, M. Petric ${ }^{d}$, M. Žitnik ${ }^{d}$, K. Kvashnina ${ }^{e}$, G. B. M. Vaughan $^{e}$, R. Bouchet ${ }^{b}, F$. Alloin $^{b, c}$, and M. Kavčic ${ }^{d, f^{*}}$

${ }^{\text {a } U n i v . ~ G r e n o b l e ~ A l p e s, ~ C E A, ~ L I T E N, ~ D E H T, ~ S T B, ~} 38000$ Grenoble, France

${ }^{\mathrm{b}}$ Univ. Grenoble Alpes, Univ. Savoie Mont Blanc, CNRS, Grenoble INP, LEPMI, 38000 Grenoble, France

${ }^{\mathrm{c}}$ Réseau sur le Stockage Electrochimique de 1'Energie (RS2E), CNRS, FR3459, 80039 Amiens Cedex, France

d Jožef Stefan Institute, Jamova 39, 1000 Ljubljana, Slovenia

${ }^{\mathrm{e}}$ European Synchrotron Radiation Facility, 38000 Grenoble, France

${ }^{\mathrm{f}}$ Faculty of Mathematics and Physics, University of Ljubljana, Jadranska 19, 1000 Ljubljana, Slovenia 


\section{Corresponding Authors}

* Mail: celine.barchasz@cea.fr; Phone: 0033438789036

* Mail: matjaz.kavcic@ijs.si ; Phone: 0038615885266

\section{ABSTRACT}

In a lithium/sulfur $(\mathrm{Li} / \mathrm{S})$ battery, the reduction of sulfur during discharge involves a particular mechanism, where the active material successively dissolves into the electrolyte to form lithium polysulfide intermediate species $\left(\mathrm{Li}_{2} \mathrm{~S}_{\mathrm{x}}\right)$, with $\mathrm{x}$ being a function of the state of charge. In this work, sulfur K-edge Resonant Inelastic X-ray Scattering measurements were performed for the characterization of different $\mathrm{Li}_{2} \mathrm{~S}_{\mathrm{x}}$ polysulfide standard solutions. High Energy Resolution Fluorescence Detected X-ray Absorption Spectroscopy allowed clear separation the pre-edge absorption peak corresponding to terminal sulfur atoms from the main absorption peak due to internal atoms, and to evaluate quantitatively the evolution of the peak area ratio as a function of the polysulfide chain length. Results of this experimental work demonstrate that the normalized area of the pre-edge is a reliable fingerprint of $\mathrm{Li}_{2} \mathrm{~S}_{\mathrm{x}}$ mean chain length in agreement with recent theoretical predictions. As a perspective, this work confirms that operando HERFD XAS can be used to differentiate mean polysulfide composition, which is key issue in the characterization of $\mathrm{Li} / \mathrm{S}$ cells. 


\section{TOC GRAPHICS}

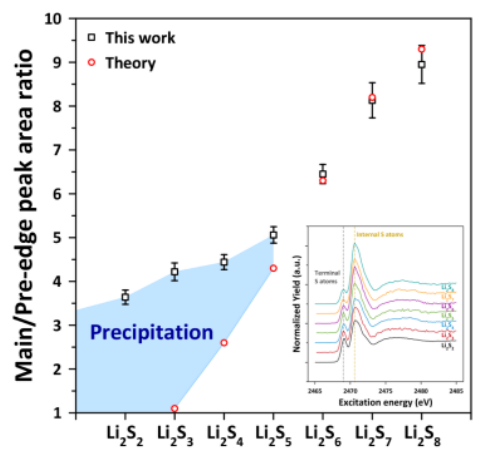

\section{KEYWORDS}

Lithium polysulfide standards; Sulfur K-edge RIXS measurements, sulfur HERFD-XAS spectra, Polysulfide mean chain length 
Lithium/sulfur $(\mathrm{Li} / \mathrm{S})$ batteries are one of the most attractive rechargeable energy storage systems, thanks to the low cost and abundance of elemental sulfur, as well as the high theoretical gravimetric energy density attainable. ${ }^{1}$ This post lithium-ion system usually relies on the use of a lithium metal electrode, an organic electrolyte and a sulfur based cathode where the elemental sulfur $\left(\mathrm{S}_{8}\right)$ is reduced to produce solid $\mathrm{Li}_{2} \mathrm{~S}$. The electrochemical activity of sulfur (or of the lithiated $\mathrm{Li}_{2} \mathrm{~S}$ counterpart) involves a particular cycling mechanism, where the active material successively dissolves into the electrolyte with the formation of lithium polysulfide (PS) intermediate species $\left(\mathrm{Li}_{2} \mathrm{~S}_{\mathrm{x}}, 2 \leq \mathrm{x} \leq 8\right)$, with $\mathrm{x}$ being a function of the stateof-charge, and precipitates at the positive electrode $\left(\mathrm{S}_{8}\right.$ and $\left.\mathrm{Li}_{2} \mathrm{~S}\right)$. Thus, the electrochemical processes of $\mathrm{Li} / \mathrm{S}$ batteries are complex, and still subject to debate.

Different discharge mechanisms have already been proposed, involving different lithium PS intermediate species and reaction pathways. ${ }^{2,3,4,5}$ However, the complex chemical and electrochemical equilibria, as well as the lack of analytical techniques to unambiguously determine the exact composition of $\mathrm{Li}_{2} \mathrm{~S}_{\mathrm{x}}$, make the determination of a univocal discharge mechanism very difficult. High-performance liquid chromatography has been applied ex situ, and offers a good selectivity towards each polysulfide. ${ }^{2,3}$ However, the detailed understanding of the discharge mechanism requires the use of operando methods, with both good chain length selectivity and time resolution. To achieve this, operando UV-vis and X-ray Absorption (XAS) spectroscopy techniques have been applied to Li/S cells, providing partial information on $\mathrm{Li}_{2} \mathrm{~S}_{\mathrm{X}}$ intermediate species. ${ }^{3,4,5,6}$ In particular, operando X-Ray Absorption Near Edge Structure (XANES) spectroscopy, which is capable of detecting both solid and liquid phases, has been successfully applied in several Li/S battery studies, ${ }^{5,7,8}$ giving information relative to the oxidation state of sulfur species including $\mathrm{Li}_{2} \mathrm{~S}_{\mathrm{x}}$ during the battery cycle. 
A linear combination fitting analysis was used in these studies to extract relative amounts of the three main sulfur compounds formed in the battery. Reference spectra used in the fit were either obtained by measuring $\mathrm{Li}_{2} \mathrm{~S}_{\mathrm{x}}$ standards or $\mathrm{Na}_{2} \mathrm{~S}_{\mathrm{x}}$ substitutes ${ }^{5}$, or in some cases extracted directly from a set of operando spectra ${ }^{7}$. While the total relative amount of polysulfides produced within the battery could be followed during the cycle, it was only possible to make a rough qualitative estimate of the polysulfide chain length. However, a recent theoretical study $^{15}$ suggested a linear relationship between the peak areas of the two main spectral features and the polysulfide chain length, which could be used to differentiate the polysulfides from XANES spectra. In such measurements, the energy resolution of the measured absorption spectra is ultimately limited by the core-hole lifetime broadening and this represents an obstacle in resolving clearly the main features in the $\mathrm{Li}_{2} \mathrm{~S}_{\mathrm{x}}$ absorption spectrum. Compared to standard fluorescent mode XAS measurements, energy resolution can be enhanced below the core-hole lifetime broadening using Resonant Inelastic X-ray Scattering (RIXS). In this case, the fluorescence is detected by the emission spectrometer with energy resolution comparable to the resolution of the beamline monochromator. The overall spectral broadening is in this case limited mainly by the experimental resolution (the final state broadening is usually almost negligible), and a significant sharpening effect can be observed in the RIXS spectra. While RIXS in the hard X-ray range is commonly exploited to study $3 \mathrm{~d}$ transition complexes ${ }^{9}$, the tender X-ray range containing the sulfur absorption edge has been much less explored, mainly due to several technical challenges inherent to measurements in this low energy range.

In our previous work, ${ }^{10}$ operando RIXS measurements at the sulfur K-edge were successfully applied to enhance the sensitivity for lithium polysulfide detection, by exciting the polysulfides resonantly compared to the solid $\mathrm{S}_{8}$ and $\mathrm{Li}_{2} \mathrm{~S}$ counterparts. In the present work, sulfur K-edge RIXS measurements were used for the characterization of different $\mathrm{Li}_{2} \mathrm{~S}_{\mathrm{X}}$ 
polysulfides. In order to closely mimic the environment in a $\mathrm{Li} / \mathrm{S}$ cell, measurements were performed on dissolved lithium PS standard solutions. Enhanced spectral resolution was used to decompose the main spectral features and determine precisely their area ratios, which can be directly compared to theoretical predictions. ${ }^{15}$

An example of a full K-L RIXS map is shown in Fig. 1(a) for the $\mathrm{Li}_{2} \mathrm{~S}_{2}$ equivalent standard solution. The map was built by recording a series of 100 consecutive sulfur $\mathrm{K} \alpha$ emission spectra while changing the excitation energy across the sulfur $\mathrm{K}$ absorption edge in $0.2 \mathrm{eV}$ steps. Due to the high experimental energy resolution, a significant narrowing can be observed in the measured RIXS map, with the first pre-edge resonance due to terminal sulfur atoms well resolved from the main absorption peak corresponding to internal sulfur atoms. By making a horizontal cut through the measured RIXS map at fixed emission energy corresponding to the top of the $\mathrm{K} \alpha 1$ emission line, an absorption like spectrum with superior resolution is obtained. This technique is referred as High Energy Resolution Fluorescence Detected XAS (HERFD-XAS), ${ }^{11,12}$ and can be used to overcome the intrinsic core-hole lifetime broadening limitation of the XAS spectroscopy. This is demonstrated in Fig. 1(b), comparing the measured $\mathrm{Li}_{2} \mathrm{~S}_{2}$ sulfur K-edge HERFD-XAS spectrum with the standard XAS spectrum measured as well. Significant improvement in spectral resolution is observed in Fig. $1(b)$. 

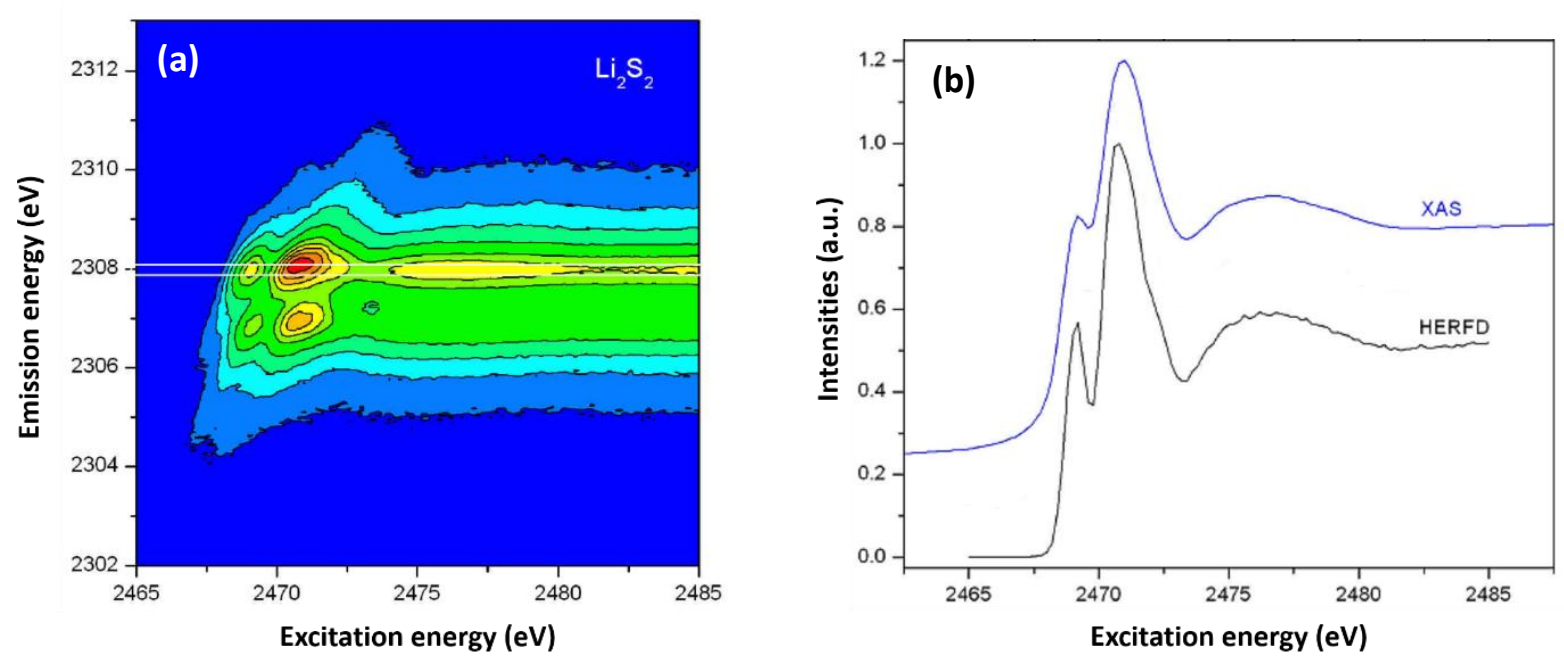

Figure 1. RIXS map of $\mathrm{Li}_{2} \mathrm{~S}_{2} 10^{-1}$ mol. $\mathrm{L}^{-1}$ standard solution in TEGDME/DIOX 1/1. The vertical axis corresponds to the emission energy around K-L $\left(\mathrm{K}_{\alpha}\right)$ transition and horizontal axis corresponds to the excitation energy around the $\mathrm{K}$ absorption edge of sulfur (a). Comparison of experimental spectra obtained by standard XAS (in blue) and by horizontal cut through the RIXS map (in black) (b).

Compared to standard XAS, the increased resolution of HERFD XAS spectra allows the preedge peak attributed to terminal atoms of lithium PS $(\sim 2469 \mathrm{eV})$ to be clearly resolved from the main peak $(\sim 2471 \mathrm{eV})$ generally attributed to elemental sulfur ( $\mathrm{S}-\mathrm{S}$ bond in $\mathrm{S}_{8}$ ring). ${ }^{10,18,13,14,15}$ Observation of Fig. 2 shows as predicted that the relative area (resp. intensity) of the pre-edge peak increases while the polysulfide chain length decreases. Quantitative analysis can be performed by determining the area (resp. intensity) of the pre-edge peak relative to that of the main one, to better differentiate the polysulfide species. 


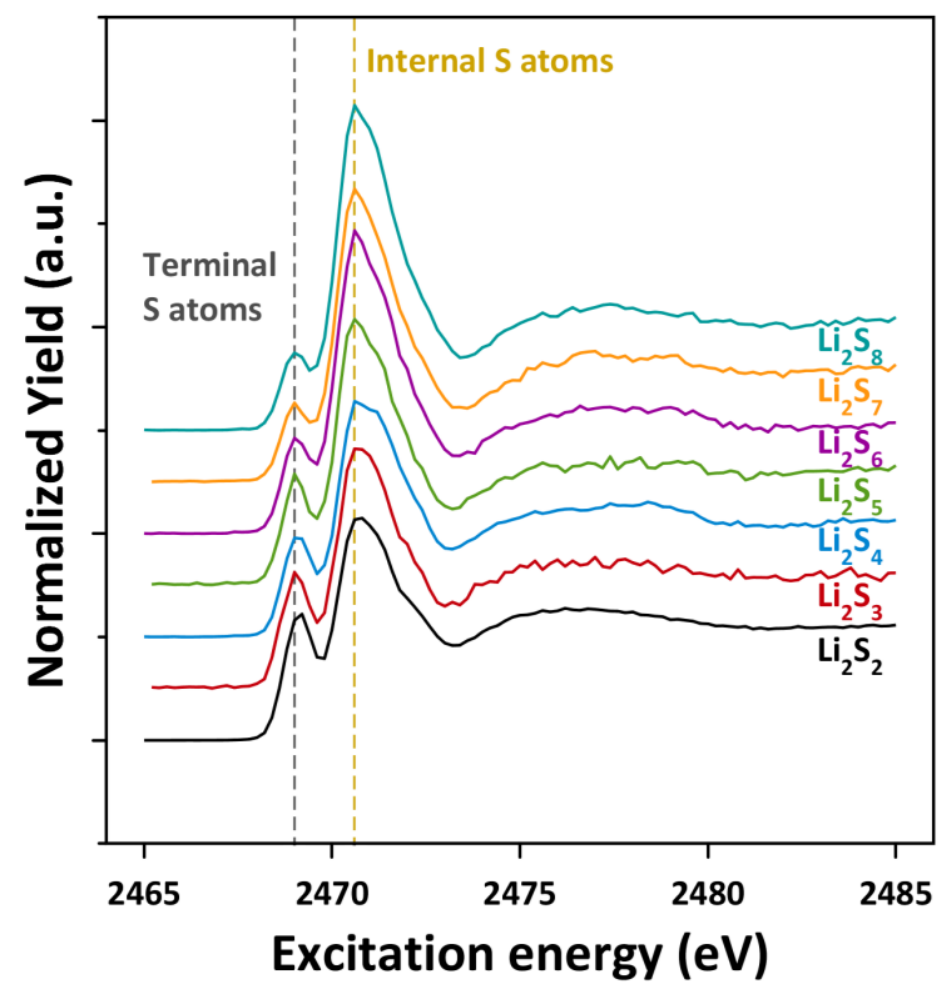

Figure 2. Normalized HERFD sulfur K-edge absorption spectra recorded for different lithium PS standard solutions $\operatorname{Li}_{2} \mathrm{~S}_{\mathrm{x}}(\mathrm{x}=2 \ldots 8)$ at $10^{-1}$ mol. $\mathrm{L}^{-1}$ in TEGDME/DIOX 1/1. The spectra are shifted vertically for clarity.

In a recent comprehensive theoretical work of Pascal et al., ${ }^{15}$ sulfur XAS spectra of lithium PS dissolved in TEGDME were simulated using first-principles molecular dynamics calculations. Their calculations confirm that the two main absorption features arise from differently coordinated sulfur atoms in the polysulfide molecule. The pre-edge feature is attributed to terminal sulfur atoms associated to lithium atoms at either end of the polysulfide and the main-edge to the internal sulfur atoms. Density functional theory (DFT) calculations revealed increased local electron density (charge) of the terminal atoms compared to the internal ones, leading to reduced binding energy of the core electron and consequently the shift of the corresponding absorption feature to lower energies with respect to those corresponding to internal atoms in the polysulfide chain. This attribution of main absorption 
features was confirmed also by the work of Kosugi et al., ${ }^{16}$ who have carried out ab initio calculations on XAS spectrum of $\mathrm{S}_{2}$. An almost linear relationship between the normalized peak areas (resp. intensities) of the two main absorption features and the PS chain length was found by Pascal et al. ${ }^{15}$ and this is suggested to be used to resolve different PS from XAS spectrum.

In order to check these theoretical predictions, the measured HERFD XAS spectra were fit to the model consisting of both main absorption features followed by an edge. A single Gaussian was used for the pre-edge absorption peak. Due to the high experimental energy resolution, the main absorption peak reveals a slight asymmetry on the high energy side, which can be accounted for by adding two additional arbitrary Gaussians on the high energy tail of the main Gaussian peak. A cumulative Gaussian distribution was used to describe the edge. This model describes well the measured spectra and is used to extract peak area (and intensity) ratios and energy splitting of the two main absorption peaks (Fig. 3 a).

The experimental values can be directly compared to the theoretical predictions of Pascal et $a l .{ }^{15} \mathrm{~A}$ comparison of the peak area ratios as a function of PS mean chain length is presented in Fig. 3 b. Very good agreement is obtained for long chain PS $\operatorname{Li}_{2} \mathrm{~S}_{\mathrm{x}}(\mathrm{x} \geq 6)$, confirming the linear slope predicted by the theory. For shorter chain PS, this slope is less pronounced and the difference between experimental and theoretical values increases. For the shortest $\mathrm{Li}_{2} \mathrm{~S}_{2}$ PS sample, the experimental spectrum still exhibits both absorption features, whereas only the pre-edge peak due to sulfur external atoms should be observed, as no internal sulfur atoms exist in this shortest polysulfide. This difference is attributed to the fact that the standard solutions do not consist of a pure $\mathrm{Li}_{2} \mathrm{~S}_{\mathrm{x}}$ species, but of lithium PS mixtures in equilibrium with a mean composition $\mathrm{Li}_{2} \mathrm{~S}_{\mathrm{x}}$, with the possible presence of precipitated species. ${ }^{3}$ Indeed, it is well known that lithium PS in solution tend to disproportionate and comproportionate in rapid equilibrium. The presence of chemical equilibria in PS solutions, associated with 
disproportionation reactions, ${ }^{3}$ is responsible for the formation of multi PS species in one mean $\mathrm{Li}_{2} \mathrm{~S}_{\mathrm{x}}$ composition. Despite that, the ratio of internal/terminal sulfur atoms in solution, which is the experimental quantity determined by HERFD-XAS, is still conserved and reflects the mean PS chain length corresponding to the nominal $x$ value of the initial $\mathrm{Li}_{2} \mathrm{~S}_{\mathrm{X}}$ standard solution. On the other hand, such chemical equilibria have an indirect impact on the shorter chains solutions, due to the formation of solid species and/or the incomplete reaction of $\mathrm{Li}$ with $\mathrm{S}_{8}$. Indeed, $\mathrm{Li}_{2} \mathrm{~S}$ (and possibly $\mathrm{Li}_{2} \mathrm{~S}_{2}$ ) being less soluble than other PS counterparts, ${ }^{3}$ the disproportionation reactions lead to the formation of insoluble products, which precipitate from the solution. ${ }^{17}$ Due to the precipitation of these species, experimental HERFD spectra correspond to $\mathrm{Li}_{2} \mathrm{~S}_{\mathrm{x}}$, solutions, with mean chain length $\mathrm{x}$ ' larger than the nominal $\mathrm{x}$ value of the short chain PS standard solutions. While the absence of solid species due to the high solubility of PS enables the theoretically predicted ratios to be obtained for long PS chains, the precipitation of less soluble short PS chains explains the discrepancy between experimental values and theory, which increases with decreasing the PS chain length.
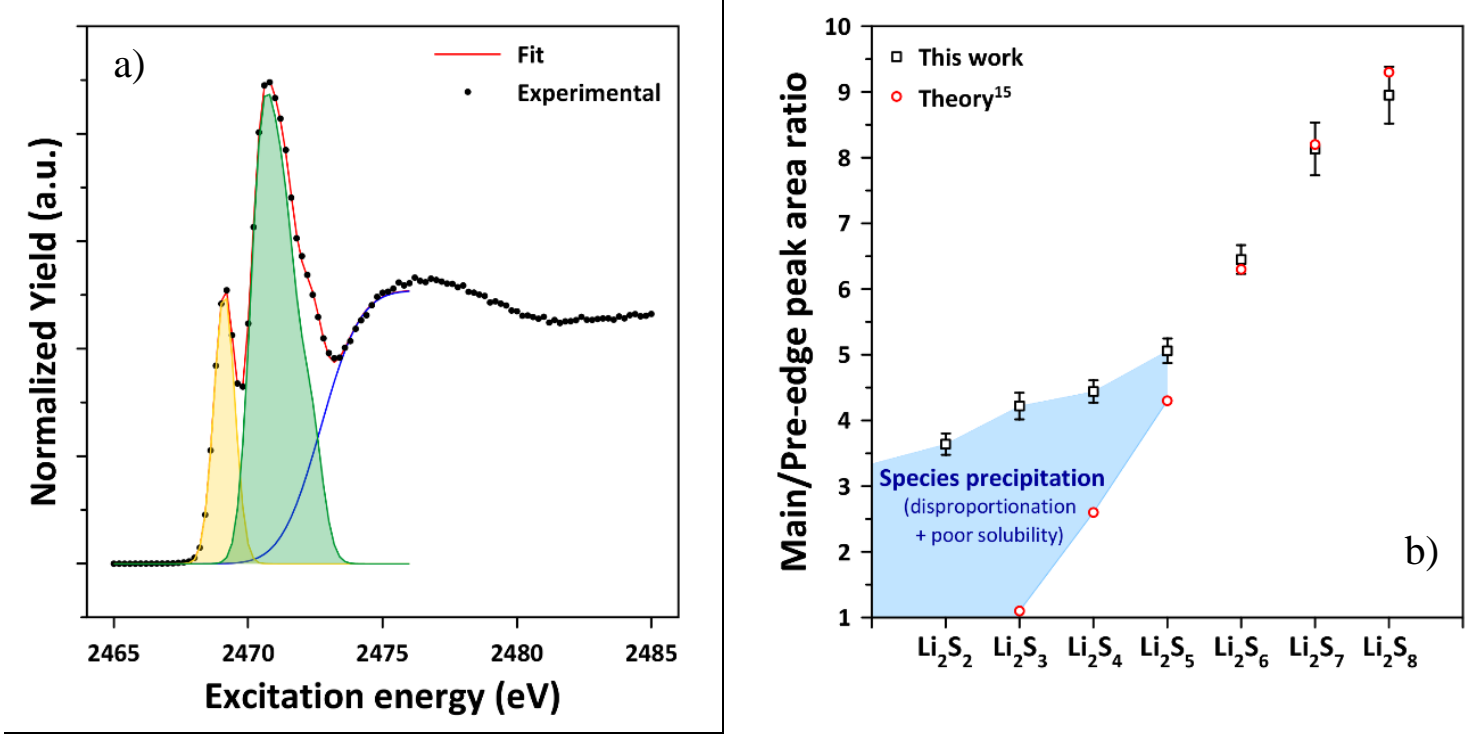

Figure 3. (a) HERFD sulfur K-edge absorption spectrum recorded for the $\mathrm{Li}_{2} \mathrm{~S}_{2}$ standard solution fitted with the model described in the text. (b) Experimental area ratios for the two absorption peaks compared to theoretical values of Pascal et al. ${ }^{15}$ 
In addition to peak area ratios, the terminal/internal peak splitting have also been extracted from the spectra yielding an average value of $1.46 \pm 0.05 \mathrm{eV}$ for all of the standard PS solutions. This is in agreement with the theoretically predicted $1.5 \mathrm{eV}$ value for the longest $\mathrm{Li}_{2} \mathrm{~S}_{8}$ polysulfide chain. ${ }^{15}$ According to theoretical calculations, a correlation between peak splitting and partial atomic charge difference is expected so that the splitting should increase to $2.1 \mathrm{eV}$ approaching $\mathrm{Li}_{2} \mathrm{~S}_{3}$. However, no significant variation of peak splitting between different measured standards has been observed in the present experiment. Despite significant discrepancies of experimental peak area ratios from the theorical values observed for short chain PS, at least a slight systematic variation of the peak splitting as a function of the PS chain length would be expected, but this is not supported by our experimental data.

To conclude, the increased spectral resolution of HERFD sulfur K-edge absorption spectra of standard PS samples has allowed the clear separation of the pre-edge absorption peak corresponding to terminal sulfur atoms from the main absorption peak due to internal atoms. This allows the quantitative evaluation of the evolution of the peak area ratio as a function of the PS mean chain length. Results of this experimental work generally confirm the theoretical predictions of Pascal et al. that the normalized area of the pre-edge is a reliable fingerprint of $\mathrm{Li}_{2} \mathrm{~S}_{\mathrm{x}}$ mean chain length. Similar to standard XAS, HERFD XAS can be performed in operando mode, which opens the perspective to use such information to determine and quantify the soluble intermediate species formed in the battery, complementary to X-ray diffraction, which is principally used to quantify the solids formed during the electrochemical process. Therefore, a complete quantitative analysis of sulfur compounds (liquid and solid phases) formed during the electrochemical process is now feasible.

\section{EXPERIMENTAL METHODS}


Lithium polysulfide (PS) standard solutions were prepared in an argon-filled glovebox by mixing elemental sulfur (dried, 325 mesh, Alfa Aesar) and metallic lithium in a mixture of tetraethylene glycol (TEGDME, dried, 99\%, Adrich) and 1,3-dioxolane (DIOX, anhydrous, $99,8 \%$, Aldrich) with a volume ratio of $1 / 1$. The different lithium PS standard solutions, with equivalent compositions of $\mathrm{Li}_{2} \mathrm{~S}_{\mathrm{x}}(\mathrm{x}=2,3, \ldots, 8)$, were prepared at $10^{-1} \mathrm{~mol} . \mathrm{L}^{-1}$, by using stoichiometric amounts of lithium and sulfur powders. The dispersions reacted at $50^{\circ} \mathrm{C}$ in a closed tube over a few days to ensure stable composition of the solutions.

For the purpose of RIXS measurements, lithium PS standard solutions were enclosed in a CR2032 coin cell designed for XAS experiments. The coin cells were assembled in an argon filled glovebox, the casing was drilled on one side and airtightness was achieved using a Kapton $^{\circledR}$ film $(7 \mu \mathrm{m})$, in order to allow X-rays to reach the sample and collect the fluorescence signal.

Measurements were performed at the ID26 beamline of the European Synchrotron Radiation Facility (ESRF) equipped with a double $\mathrm{Si}(111)$ monochromator providing an energy resolution of $\sim 0.35 \mathrm{eV}$ at the sulfur $\mathrm{K}$-edge $(2472 \mathrm{eV})$. The photon beam was focused to $50 \times$ $250 \mu \mathrm{m}$ and the incoming flux was $\sim 5 \times 10^{12}$ photons/s. RIXS spectra were recorded by an in-vacuum Johansson type curved-crystal spectrometer for high-energy resolution X-ray emission spectroscopy in the tender range. ${ }^{18}$ The spectrometer operates in the dispersive mode using CCD camera containing $770 \times 1152$ pixels with $22.5 \mu \mathrm{m}^{2}$ pixel. The first order reflection of the $\mathrm{Si}(111)$ analyzer crystal was used for this experiment. At fixed detector position tuned to central Bragg angle corresponding to the $\mathrm{S} \mathrm{K} \alpha$ emission line the spectrometer covered the full bandwidth of $\sim 29 \mathrm{eV}$ with energy resolution of $\sim 0.4 \mathrm{eV}$. In addition, a Si photodiode was mounted in the spectrometer chamber to collect also fast XANES spectra, which were used to monitor any possible radiation damage induced by the incident photon beam. 


\section{AUTHOR INFORMATION}

The authors declare no competing financial interests.

\section{ACKNOWLEDGMENT}

The authors acknowledge UGA (Université Grenoble Alpes) for Ph.D funding awarded to Alice Robba and thank ESRF for allocation of beamtime and ID26 beamline staff for their assistance during experiment. Work has been supported by the Slovenian Research Program P1-0112. 


\section{REFERENCES}

(1)Manthiram A., Fu Y., Chung S.-H., Zu C., Su Y.-S., Rechargeable Lithium-Sulfur Batteries, Chem. Rev. 2014, 114, 11751-11787

(2)Zheng D., Liu D., Harris J. B., Ding T., Si J., Andrew S., Qu D., Yang X.-Q., Qu D., Investigation of the Li-S Battery Mechanism by Real-Time Monitoring of the Changes of Sulfur and Polysulfide Species during the Discharge and Charge, ACS Appl. Mater. Interfaces 2017, 9, 4326-4332

(3) Barchasz, C. Molton F., Duboc C., Leprêtre J.-C., Patoux S., Alloin F., Lithium/Sulfur Cell Discharge Mechanism: An Original Approach for Intermediate Species Identification, Anal. Chem. 2012, 84, 3973-3980

(4) Dominko R., Vizintin A., Aquilanti G., Stievano L., Helen M. J., Munnangi A. R., Fichtner M., Arcon I., Polysulfides Formation in Different Electrolytes from the Perspective of X-ray Absorption Spectroscopy, J. Electrochem. Soc., 2018, 165 A5014-A5019

(5) Cuisinier M., Cabelguen P.-E., Evers S., He G., Kolbeck M., Garsuch A., Bolin T., Balasubramanian M., Nazar L. F., Sulfur Speciation in Li-S Batteries Determined by Operando X-ray Absorption Spectroscopy, J. Phys. Chem. Lett. 2013, 4, 3227-3232

(6) Patel M. U. M., Dominko R., Application of In Operando UV/Vis Spectroscopy in Lithium-Sulfur Batteries, ChemSusChem 2014, 7, 2167-2175

(7) Dominko, R., Patel M. U. M., Lapornik V., Vizintin A., Koželj M., Tušar N. N., Arčon I., Stievano L., Aquilanti G., Analytical Detection of Polysulfides in the Presence of Adsorption Additives by Operando X-ray Absorption Spectroscopy, J. Phys. Chem. C 2015, 119, 1900119010 
(8) Vizintin, A., Chabanne L., Tchernychova E., Arčon I., Stievano L., Aquilanti G., Antonietti M., Fellinger T.-P., Dominko, R., The mechanism of $\mathrm{Li}_{2} \mathrm{~S}$ activation in lithiumsulfur batteries: Can we avoid the polysulfide formation? J. Power Sources 2017 344, 208217

(9) Glatzel P., Bergmann U., High resolution 1s core hole X-ray spectroscopy in 3d transition metal complexes-electronic and structural information, Coord. Chem. Rev. 2005, 249, 65-95

(10) Kavčič, M., Bučar K., Petric M., Žitnik M., Arčon I., Dominko R., Vizintin A., Operando Resonant Inelastic X-ray Scattering: An Appropriate Tool to Characterize Sulfur in Li-S Batteries, J. Phys. Chem. C, 2016, 120, 24568-24576

(11) Hämäläinen K., Siddons D. P., Hastings J. B., Berman L. E., Elimination of the InnerShell Lifetime Broadening in X-ray Absorption Spectroscopy, Phys. Rev. Lett., 1991, 67, $2850-2853$

(12) Glatzel P., Juhin A., X-ray Absorption and Emission Spectroscopy, in Local Structural Characterization, D. W. Bruce, D. O’Hare, R.I. Walton (editors); John Wiley \& Sons (2013)

(13) Deslattes, R. D., Kessler E.G., Indelicato P., de Billy L., X-ray transition energies: new approach to a comprehensive evaluation, Rev. Mod. Phys., 2003, 75, 35-99

(14) Gorlin, Y., Patel M. U. M., Freiberg A., He Q., Piana M., Tromp M., Gasteiger H. A., Understanding the Charging Mechanism of Lithium-Sulfur Batteries Using Spatially Resolved Operando X-Ray Absorption Spectroscopy, J. Electrochem. Soc., 2016, 163, A930A939

(15) Pascal T. A., Wujcik K. H., Velasco-Velez J., Wu C., Teran A. A., Kapilashrami M., Cabana J., Guo J., Salmeron M., Balsara N., Prendergast D., X-ray Absorption Spectra of 
Dissolved Polysulfides in Lithium-Sulfur Batteries from First-Principles, J. Phys. Chem. Lett., 2014, $5,1547-1551$

(16) Rühl, E., Flesch, R., Tappe, W., Novikov, D. Kosugi, N. Sulfur 1s excitation of $S_{2}$ and $\mathrm{S}_{8}$ : Core-valence- and valence-valence-exchange interaction and geometry-specific transitions, J. Chem. Phys. 2002, 116, 3316-3322

(17) Talian S. D., Kapun G., Moškon J., Vizintin A., Randon-Vitanova A., Dominko R., Gaberšček M., Which Process Limits the Operation of a Li-S System?, Chem. Mater., 2019, $31,9012-9023$

(18) Kavčič, M., Budnar M, Mühleisen A, Gasser F, Žitnik M, Bučar K, Bohinc R., Design and performance of a versatile curved-crystal spectrometer for high-resolution spectroscopy in the tender X-ray range, Rev. Sci. Instrum., 2012, 83, 1-8 